ИЗВЕСТИЯ АКАДЕМИИ НАУК ЭСТОНСКОП ССР. ФИЗИКА * МАТЕМАТИКА

PROCEEDINGS OF THE ACADEMY OF SCIENCES OF THE ESTONIAN SSR. PHYSICS * MATHEMATICS

$1986,35,3$

удК 535.33

Инна РЕБАНЕ

\title{
К ТЕОРИИ ДВУХСТУПЕНЧАТОГО ФОТОВЫЖИГАНИЯ СПЕКТРАЛЬНОГО ПРОВАЛА
}

\author{
(Представил В. Хижняков)
}

Метод фотовыжигания провала (ФВП) $\left[{ }^{1,2}\right]$ находит все более широкое применение в спектроскопии твердого тела [ $\left.{ }^{3,4}\right]$. Наряду со стационарным выжиганием узкой линией непрерывного лазера стали применяться и световые импульсы $\left[{ }^{5}\right]$. В [ $\left.{ }^{6}\right]$ показано, что при одноступенчатом фотовыжигании получаемый спектральный провал одинаков как при выжигании одиночным импульсом, так и при стационарном выжигании при условии, что дозы выжигания (т. е. суммарное за все время воздействия света на фотохромную пленку число фотонов) на каждой частоте одинаковы.

Наряду с одноступенчатыми процессами выжигания внимание исследователей начинают привлекать и двухступенчатые процессы '[7,8 $]$, в которых фототрансформация происходит в результате последовательного поглощения двух фотонов и которые становятся особо эффективными при импульсном возбуждении. Двухступенчатое выжигание открывает новые возможности перед спектроскопией фотовыжигания и его практическими приложениями, заодно порождая и новые теоретические проблемы.

Целью данной работы является рассмотрение двухступенчатого выжигания провала в функции неоднородного распределения частот оптических переходов в трехуровневой системе двумя следующими друг за другом импульсами.

Моделируем процесс фотовыжигания следующим образом. Рассмотрим ансамбль трехуровневых систем $\{0,1,2\}$ с неоднородным распределением $\mathrm{Q}\left(\Omega_{01}, \Omega_{12}, t\right)$ энергий $\Omega_{01}$ и $\Omega_{12}$ переходов $0 \rightarrow 1$ и $1 \rightarrow 2$ соответственно. Вследствие взаимодействия с двумя световыми импульсами, содержащими резонансные с этими переходами частоты (первый импульс в резонансе с переходом $0 \rightarrow 1$, а не с $2 \rightarrow 2$, второй - в резонансе только с переходом $1 \rightarrow 2$ ), система из состояния 0 последовательно переходит в первое возбужденное состояние 1 и затем во второе возбужденное состояние 2. Далее, в состоянни 2 с вероятностью $\alpha$ происходит превращение возбужденной системы, в результате чего она перестает поглощать на прежней частоте $\Omega_{01}$ перехода $0 \rightarrow 1$, а также на частоте $\Omega_{12}$ перехода $1 \rightarrow 2$.

В результате прохождения световых импульсов через селективную фотохромную среду функция неоднородного распределения энергии $\left[{ }^{3}\right]$ оптических переходов трехуровневых систем будет меняться во времени по закону

$$
\mathrm{Q}\left(\Omega_{01}, \Omega_{12}, t\right)=\mathrm{Q}_{0}\left(\Omega_{01}, \Omega_{12}\right)\left[1-P\left(\Omega_{01}, \Omega_{12}, t\right)\right],
$$


Где $\varrho_{0}\left(\Omega_{01}, \Omega_{12}\right)$ - первоначальная функция неоднородного распреде ления. $P\left(\Omega_{01}, \Omega_{12}, t\right)$ - вероятность того, что к моменту времени $t$ сисгема перестанет поглощать частоты $\Omega_{01}$ и $\Omega_{12}$. При условии достаточно малых доз облучения можно обеспечить выполнимость условия $P\left(\Omega_{01}, \Omega_{12}, t\right) \ll 1$ и рассмотреть процесс образования провала во втором порядке теории возмущений, учитывая только один акт последовательного поглощения двух фотонов.

Рассмотрим следующее начальное состояние возбуждающего (выжигающего) поля $\left(t_{0} \rightarrow-\infty\right)$ :

$$
\left|\psi_{R}\left(t_{0}\right)\right\rangle=\iint d_{\omega_{1}} d_{\omega_{2}} g\left(\omega_{1}, \omega_{2}\right)\left|\omega_{1}\right\rangle\left|\omega_{2}\right\rangle \exp \left[-i\left(\omega_{1}+\omega_{2}\right) t_{0}\right]
$$

с условиями резонанса $\omega_{1} \approx \Omega_{01}, \omega_{2} \approx \Omega_{12}\left(\Omega_{01} \neq \Omega_{12}\right)$, т. е. частота первого (в общем случае частоты пакета) возбуждения находится в полосе переходов $\Omega_{01}$, второго $-\Omega_{12}$, считается, что перекрывания полос $\Omega_{01}$ и $\Omega_{12}$ нет. Тогда в случае начального состояния $(2)$, используя теорию зависящего от времени резонансного вторичного свечения $\left[{ }^{9,10}\right]$ вероятность $P\left(\Omega_{01}, \Omega_{12}, t\right)$ определяется следующей формулой:

$$
\begin{gathered}
P\left(\Omega_{01}, \Omega_{12}, t\right)=\alpha \int_{-\infty}^{t} d t^{\prime} \iiint \int d \omega_{1} d \omega_{2} d \omega_{1}^{\prime} d \omega_{2}^{\prime} g\left(\omega_{1}, \omega_{2}\right) g^{*}\left(\omega_{1}^{\prime}, \omega_{2}^{\prime}\right) \times \\
\times \iint_{-\infty}^{t^{\prime}} d t_{1} d t_{1}^{\prime} \int_{-\infty}^{t_{1}} d t_{2} \int_{-\infty}^{t_{1}^{\prime}} d t_{2}^{\prime} F\left(t^{\prime}, t_{1}, t_{1}^{\prime}, t_{2}, t_{2}^{\prime}\right) \exp \left[-i\left(\omega_{1} t_{2}+\omega_{2} t_{1}\right)\right] \times \\
\times \exp \left[i\left(\omega_{1}^{\prime} t_{2}^{\prime}+\omega_{2}^{\prime} t_{1}^{\prime}\right] .\right.
\end{gathered}
$$

В формуле (3) $\alpha$ - вероятность превращения возбужденной на уровень 2 системы, $F\left(t^{\prime}, t_{1}, t^{\prime}{ }_{1}, t_{2}, t^{\prime}{ }_{2}\right)$ - корреляционная функция трехуровневой системы.

Вероятность $P\left(\Omega_{01}, \Omega_{12}, t\right)$ можно записать также в виде

$$
\begin{gathered}
P\left(\Omega_{01}, \Omega_{12}, t\right)=\alpha \int_{-\infty}^{t} d t^{\prime} \iint_{-\infty}^{t^{\prime}} d t_{1} d t_{1}^{\prime} \int_{-\infty}^{t_{1}} d t_{2} \int_{-\infty}^{t_{1}^{\prime}} d t_{2}^{\prime} g\left(t_{2}, t_{1}\right) \times \\
\times g^{*}\left(t_{2}^{\prime}, t_{1}^{\prime}\right) F\left(t^{\prime}, t_{1}, t_{1}^{\prime}, t_{2}, t_{2}^{\prime}\right),
\end{gathered}
$$

где

$$
g\left(x_{1}, x_{2}\right)=\iint d \omega_{1} d \omega_{2} g\left(\omega_{1}, \omega_{2}\right) \exp \left[-i\left(\omega_{1} x_{1}+\omega_{2} x_{2}\right)\right] .
$$

Используем в (3) новые переменные

$$
\begin{array}{ll}
\mu=t_{1}^{\prime}-t_{1}, & \tau=t_{1}^{\prime}-t_{2}^{\prime}, \\
\nu=t^{\prime}-\frac{1}{2}\left(t_{1}+t_{1}^{\prime}+|\mu|\right), & \tau^{\prime}=t_{1}-t_{2} .
\end{array}
$$

\section{Получим}

$$
P\left(\Omega_{01}, \Omega_{12}, t\right)=2 \alpha \operatorname{Re}\left\{\int_{-\infty}^{t} d t^{\prime} \iiint \int d \omega_{1} d \omega_{2} d \omega_{1}^{\prime} d \omega_{2}^{\prime} g\left(\omega_{1}, \omega_{2}\right) \times\right.
$$

$\times g^{*}\left(\omega_{1}^{\prime}, \omega_{2}^{\prime}\right) \iiint_{0}^{\infty} \int d \mu d v d \tau d \tau^{\prime} F\left(\mu, v, \tau, \tau^{\prime}\right) \exp \left[-i \omega_{1}\left(t^{\prime}-\mu-v-\tau^{\prime}\right)\right] \times$

$$
\left.\times \exp \left[i \omega_{1}^{\prime}\left(t^{\prime}-v-\tau\right)-i \omega_{2}\left(t^{\prime}-\mu-v\right)+i \omega_{2}^{\prime}\left(t^{\prime}-v\right)\right]\right\}
$$


Пॉерейдя к пределу $t \rightarrow \infty$, получим

$$
\begin{gathered}
P\left(\Omega_{01}, \Omega_{12}\right) \equiv \lim _{t \rightarrow \infty} P\left(\Omega_{01}, \Omega_{12}, t\right)=2 \alpha \operatorname{Re}\left\{\iiint d \omega_{1} d \omega_{2} d \omega_{1}^{\prime} g\left(\omega_{1}, \omega_{2}\right) \times\right. \\
\times g^{*}\left(\omega_{1}^{\prime}, \omega_{1}+\omega_{2}-\omega_{1}^{\prime}\right) \iiint \int d \mu d v d \tau d \tau^{\prime} F\left(\mu, v, \tau, \tau^{\prime}\right) \times \\
\left.\times \exp \left[i \omega_{1}\left(\mu+\tau^{\prime}\right)-i_{\omega_{1}^{\prime}} \tau+i_{\omega_{2} \mu}\right]\right\} .
\end{gathered}
$$

Вероятность возбуждения (выжигания) $P\left(\Omega_{01}, \Omega_{12}\right)$ с помощью формулы (1) определяет получаемый спектральный провал при выжигании импульсами.

Для сравнения приведем вероятность $P_{\text {ст }}\left(\Omega_{01}, \Omega_{12}\right)$ при произвольном стационарном возбуждении (выжигании)

$$
P_{\text {ст }}\left(\Omega_{01}, \Omega_{12}\right)=\iint d_{\omega_{1}} d_{\omega_{2}}\left|g\left(\omega_{1}, \omega_{2}\right)\right|^{2} W\left(\Omega_{01}, \Omega_{12}\right),
$$

где

$$
\begin{gathered}
W\left(\Omega_{01}, \Omega_{12}\right)=2 \alpha \operatorname{Re}\left\{\iiint_{0}^{\infty} \int d \mu d v d \tau d \tau^{\prime} F\left(\mu, v, \tau, \tau^{\prime}\right) \times\right. \\
\left.\times \exp \left[i_{\omega_{1}}\left(\mu+\tau^{\prime}-\tau\right)+i_{\omega_{2}} \mu\right]\right\}
\end{gathered}
$$

- вероятность возбуждения (выжигания) двумя монохроматическими волнами с частотами $\omega_{1}$ и $\omega_{2}$. Нетрудно видеть, что (8) сводится к (9) только в частном случае, когда $\omega_{1}^{\prime}=\omega_{1}$.

Таким образом, вероятность возбуждения (выжигания) $P\left(\Omega_{01}, \Omega_{12}\right)$, а отсюда и получаемый спектральный провал существенно различны при выжиганни импульсами и при стационарном выжигании.

Рассмотрим некоторые примеры для иллюстрации формулы (8), используя простую модель для $F\left(t^{\prime}, t_{1}, t^{\prime}{ }_{1}, t_{2}, t^{\prime}{ }_{2}\right)$

$$
\begin{gathered}
F\left(t^{\prime}, t_{1}, t_{1}^{\prime}, t_{2}, t_{2}^{\prime}\right)=C \exp \left[-\gamma_{2} t^{\prime}+i \Omega_{12}\left(t_{1}-t_{1}^{\prime}\right)+i \Omega_{01}\left(t_{2}-t_{2}^{\prime}\right)+\right. \\
\left.+\gamma_{2}\left(t_{1}+t_{1}^{\prime}\right) / 2-\gamma_{1}\left(t_{1}+t_{1}^{\prime}-t_{2}-t_{2}^{\prime}\right) / 2\right]
\end{gathered}
$$

или в переменных (6)

$$
\begin{gathered}
F\left(\mu, v, \tau, \tau^{\prime}\right)=C \exp \left[-i \Omega_{12} \mu+i \Omega_{01}\left(\tau-\tau^{\prime}-\mu\right)-\gamma_{1}\left(\tau+\tau^{\prime}\right) / 2-\right. \\
\left.-\gamma_{2}(v+|\mu| / 2)\right],
\end{gathered}
$$

где $\gamma_{1}$ и $\gamma_{2}$ константы затухания соответственно уровней 1 и 2 .

В этой модели для стационарного случая из (10) получаем

$$
W\left(\Omega_{01}, \Omega_{12}\right)=\alpha C /\left\{\left[\left(\omega_{1}-\Omega_{01}\right)^{2}+\gamma_{1}^{2} / 4\right]\left[\left(\omega_{1}+\omega_{2}-\Omega_{01}-\Omega_{12}\right)^{2}+\gamma_{2}^{2} / 4\right]\right\} .
$$

1. Сравним следующие два случая: во-первых, возбуждение (выжигание) очень короткими (дельта) импульсами соответственно с широким спектром частот и, во-вторых, стационарное возбуждение (выжигание) с широким спектром частот.

В первом случае $\quad g\left(x_{1}, x_{2}\right) \sim \delta\left(x_{1}-T_{1}\right) \delta\left(x_{2}-T_{2}\right),\left(T_{1}<T_{2}\right)$, где $T_{1}$ и $T_{2}-$ моменты времени прохождения первого и второго импульсов и $\left(t>T_{2}\right)$

$$
P(t) \sim \frac{\alpha C}{\gamma_{2}} \exp \left[-\gamma_{1}\left(T_{2}-T_{1}\right)\right]\left\{1-\exp \left[-\gamma_{2}\left(t-T_{2}\right)\right]\right\}
$$

$$
\text { и }(t \rightarrow \infty), \quad P \sim \frac{\alpha C}{\gamma_{2}} \exp \left[-\gamma_{1}\left(T_{2}-T_{1}\right)\right] .
$$


В случае стационарного выжигания $g\left(\omega_{1}, \omega_{2}\right)=$ const, и из формулы (13) следует

$$
P_{\mathrm{cT}} \sim \frac{\alpha C}{\gamma_{1} \gamma_{2}} \text {. }
$$

2. Пусть первый импульс является когерентным и затухает по экспоненциальному закону, а переход $1 \rightarrow 2$ происходит в стационарных условиях возбуждения с широким спектром частот (см. $\left.\left[{ }^{7}\right]\right)$. Тогда, записывая $g\left(t_{2}, t_{1}\right) \equiv g_{1}\left(t_{2}\right) g_{2}\left(t_{1}\right)$,

$$
g_{1}\left(t_{2}\right)=\left\{\begin{array}{lll}
0 & \text { при } & t_{2}<T_{1} \\
\varepsilon_{0} \exp \left[-i_{\omega_{10}} t_{2}-\Delta\left(t_{2}-T_{1}\right) / 2\right] & \text { при } & t_{2} \geqslant T_{1}
\end{array}\right.
$$

и $g_{2}\left(\omega_{2}\right)=$ const. В (17) $\omega_{10}-$ частота максимума, $\Delta^{-1}-$ длительность и $\Delta-$ спектральная ширина первого импульса. В этом случае

$$
\begin{aligned}
P\left(\Omega_{01}, \Omega_{12}, t\right) & =\alpha \int d \omega_{\omega_{2}}\left|g_{2}\left(\omega_{2}\right)\right|^{2} \int_{-\infty}^{t} d t^{\prime} \int_{-\infty}^{t^{\prime}} d t_{1} d t_{1}^{\prime} \int_{-\infty}^{t_{1}} d t_{2} \int_{-\infty}^{t_{1}^{\prime}} d t_{2}^{\prime} g_{1}\left(t_{2}\right) \times \\
& \times g_{1}^{*}\left(t_{2}^{\prime}\right) F\left(t^{\prime}, t_{1}, t_{1}^{\prime}, t_{2}, t_{2}^{\prime}\right) \exp \left[i_{\omega_{2}}\left(t_{1}^{\prime}-t_{1}\right)\right]
\end{aligned}
$$

и при $t \rightarrow \infty$, из формул $(11), 17)$ и (18) получаем

$$
P\left(\Omega_{01}, \Omega_{12}\right) \sim \alpha C\left|\varepsilon_{0}\right|^{2}\left(\gamma_{1}+\Delta\right) /\left\{\gamma_{1} \gamma_{2} \Delta\left[\left(\omega_{10}-\Omega_{01}\right)^{2}+\left(\gamma_{1}+\Delta\right)^{2} / 4\right]\right\} .
$$

Результат аналогичен результату фотовыжигания спектрального провала в двухуровневой системе.

3. Пусть первый импульс является когерентным и затухает по экспоненциальному закону, а второй является когерентным $\delta$-импульсом. В этом случае $\left(T_{1}<T_{2}\right)$

$$
g\left(t_{2}, t_{1}\right)= \begin{cases}0 & \text { при } t_{2}<T_{1}, \\ \varepsilon_{0} \exp \left[-i_{\omega 10} t_{2}-\Delta\left(t_{2}-T_{1}\right) / 2\right] \delta\left(t_{1}-T_{2}\right) & \text { при } t_{2} \geqslant T_{1} .\end{cases}
$$

После прохождения обоих импульсов ( $\left.t>T_{2}\right)$ из формулы (4) получаем

$$
\begin{gathered}
P\left(\Omega_{01}, t\right) \sim \alpha C\left|\varepsilon_{0}\right|^{2}\left[1-\exp \left[-\gamma_{2}\left(t-T_{2}\right)\right]\right]\left\{\exp \left[-\Delta\left(T_{2}-T_{1}\right)\right]+\right. \\
+\exp \left[-\gamma_{1}\left(T_{2}-T_{1}\right)\right]-2 \exp \left[-\left(\gamma_{1}+\Delta\right)\left(T_{2}-T_{1}\right) / 2\right] \times \\
\left.\times \cos \left[\left(\omega_{10}-\Omega_{01}\right)\left(T_{2}-T_{1}\right)\right]\right\} /\left\{\gamma_{2}\left[\left(\omega_{10}-\Omega_{01}\right)^{2}+\left(\gamma_{1}-\Delta\right)^{2} / 4\right]\right\}
\end{gathered}
$$

и $(t \rightarrow \infty)$,

$$
\begin{gathered}
P\left(\Omega_{01}\right) \sim{ }_{\alpha} C\left|\varepsilon_{0}\right|^{2}\left\{\exp \left[-\Delta\left(T_{2}-T_{1}\right)\right]+\right. \\
+\exp \left[-\gamma_{1}\left(T_{2}-T_{1}\right)\right]-2 \exp \left[-\left(\gamma_{1}+\Delta\right)\left(T_{2}-T_{1}\right) / 2\right] \times \\
\left.\times \cos \left[\left(\omega_{10}-\Omega_{01}\right)\left(T_{2}-T_{1}\right)\right]\right\} /\left\{\gamma_{2}\left[\left(\omega_{10}-\Omega_{01}\right)^{2}+\left(\gamma_{1}-\Delta\right)^{2} / 4\right]\right\} .
\end{gathered}
$$

В случае стационарного выжигания со спектральным распределе-

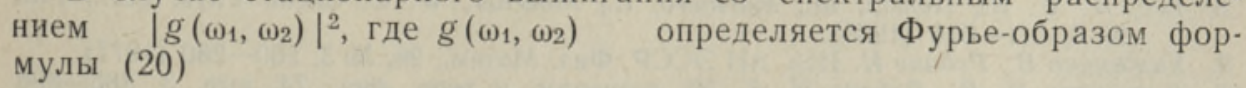

$$
\left|g\left(\omega_{1}, \omega_{2}\right)\right|^{2}=\left|\varepsilon_{0}\right|^{2} /\left[\left(\omega_{10}-\omega_{1}\right)^{2}+\Delta^{2} / 4\right],
$$

из формулы (9) получаем

$$
P_{\text {ст }}\left(\Omega_{01}\right) \sim \alpha C\left|\varepsilon_{0}\right|^{2}\left(\Delta+\gamma_{1}\right) /\left\{\gamma_{1} \gamma_{2} \Delta\left[\left(\omega_{10}-\Omega_{01}\right)^{2}+\left(\gamma_{1}+\Delta\right)^{2} / 4\right]\right\} .
$$

Таким образом, вероятности возбуждения (выжигания) $P\left(\Omega_{01}\right)$ и $P_{\text {ст }}\left(\Omega_{01}\right)$ существенно различны. Формула $(21 \mathrm{a})$ аналогична формуле, 
описывающей зависящий от времени $t$ спектр поглощения (или люминесценции) $\left[{ }^{10}\right]$, если времени $t$ сопоставить промежуток времен между импульсами $T_{2}-T_{1}$. Из формулы (2la) видно, что в данном режиме импульсного выжигания при подходящем подборе $\gamma_{1}-\Delta$ и $T_{2}-T_{1}$ получаемый провал может быть у́же, чем в стационарном режиме выжигания. Предельно узкий провал получаем при $\Delta=\gamma_{1}$ :

$$
P\left(\Omega_{01}\right) \sim \exp \left[-\gamma_{1}\left(T_{2}-T_{1}\right)\right]\left[1-\cos \left(\left(\omega_{10}-\Omega_{01}\right)\left(T_{2}-T_{1}\right)\right)\right] /\left(\omega_{10}-\Omega_{01}\right)^{2} .
$$

4. Пусть возбуждающее (выжигающее) поле определяется функцией $\left(T_{1}<T_{1}+\Delta T_{1}<T_{2}<T_{2}+\Delta T_{2}\right)$

$$
g\left(x_{1}, x_{2}\right) \sim \delta\left(x_{1}-T_{1}\right) \delta\left(x_{2}-T_{2}\right)+\delta\left(x_{1}-T_{1}-\Delta T_{1}\right) \delta\left(x_{2}-T_{2}-\Delta T_{2}\right)
$$

(нельзя представить в виде $g_{1}\left(x_{1}\right) g_{2}\left(x_{2}\right)$ ).

Тогда вероятность возбуждения (выжигания) $P(t)\left(t>T_{2}+\Delta T_{2}\right)$

$$
\begin{aligned}
P(t) \sim \frac{\alpha C}{\gamma_{2}} \exp \left[-\gamma_{1}\left(T_{2}-T_{1}-\Delta T_{1}\right)\right]\left[1-\exp \left[-\gamma_{2}\left(t-T_{2}-\Delta T_{2}\right)\right]\right] \times \\
\times\left\{\exp \left(-\gamma_{2} \Delta T_{2}-\gamma_{1} \Delta T_{1}\right)+\exp \left(-\gamma_{1} \Delta T_{2}\right)+\right. \\
\left.+2 \exp \left[-\left(\gamma_{1}+\gamma_{2}\right) \Delta T_{2} / 2-\gamma_{1} / \Delta T_{1} / 2\right] \cos \left(\Omega_{01} \Delta T_{1}+\Omega_{12} \Delta T_{2}\right)\right\},
\end{aligned}
$$

т. е. образуется двухмерная спектральная решетка. Видно, что разница $T_{2}-T_{1}$ содержится только в экспоненциальном множителе и не влияет на форму $P(t)$.

Вопросы формирования световых откликов от трехуровневых сред с преобразованной двухступенчатым выжиганием структурой двухмерной функции неоднородного распределения будут рассматриваться отдельно

Автор признателен К. К. Ребане, В. В. Хижнякову и Я. Кикасу за обсуждение работы.

\section{ЛИ ТЕРА Т У Р А}

1. Гороховский А. А., Каарли Р. К., Ребане Л. А. Письма в ЖЭТФ, 20, вып, 7, 474479 (1974); Opt. Commun., 16, № 2, 282-284 (1976).

2. Khailamov, B. M., Personov, R. I., Bykovskaya, L. A. Opt. Commun., 12, № 2, $191-193$ (1974).

3. Rebane, L. A., Gorokhouskii, A. A., Kikas, J. V. Appl. Phys. B, 29, 235-250 (1982).

4. Friedrich, J., Haarer, D. Angew. Chem., 23, № 2, 113-140 (1984) (in English).

5. Ребане А. К., Каарли Р. К., Саари П. М. Опт. и спектр., 55, вып. 3, 405-407 (1983); Письма в ЖЭТФ, 38, вып. 7, 320-323 (1983); Саари П. М., Каарли Р. К., Ребане А. К. Квантовая электроника, 12, вып. 4, 672-682 (1985).

6. Ребане И. Изв. АН ЭССР. Физ. Матем., 34, № 4, 438-440 (1985).

7. Winnacker, A., Shelby, R. M., Mcfarlane, R. M. Opt. Lett., 1985 (in press).

8. Lee, H.W. H., Gehrtz, M., Marinero, E., Moerner, W. E. Chem. Phys. Lett., 118, № $6,611-616$ (1985) .

9. Хижняков В., Ребане И. Изв. АН ЭССР. Физ. Матем., 26, № 3, 260-280 (1977).

10. Хижняков В. В., Ребане И. К. Ж. эксперим. и теор. физ,, 74, вып, 3, 885-896 (1978).

\section{Ннститут физики}

Академии наук Эстонской ССР
Поступила в редакцию 2/XII 1985 


\section{SPEKTRAALSE AUGU KAHEASTMELISE FOTOPŌLETAMISE TEOORIAST}

On vaadeldud kaheastmelist augu fotopõletamist kolmenivoolise süsteemi spektris kahe teineteisele järgneva valgusimpulsiga. Sissepõletamisel impulssidega on ergastuse (põletamise) tõenäosus ja seega ka saadav spektraalne auk oluliselt erinev statsionaarsetes tingimustes põletamisel saadavast august. On tehtud mõned mudelarvutused. Juhul kui esimeseks impulsiks on koherentne eksponentsiaalselt kustuv impulss ja teiseks $\delta$-impulss, siis sobivalt valitud esimese impulsi pikkuse ja impulssidevahelise ajavahemiku korral on võimalik saada auk, mis on kitsam statsionaarses režiimis põletamisel saadavast august.

\section{ON THE THEORY OF A TWO-STEP PHOTOBURNING} OF A SPECTRAL HOLE

A two-step photoburning of a hole in the function of the inhomogeneous distribution of optical transition frequencies in a three-level system with two successive light pulses is considered. It is shown that the probability of excitation (burning), hence the obtained spectral hole, differs essentially in the case of burning by pulses and in stationary burning conditions. Some model calculations have been made. In particular, in the case when the first pulse is coherent and decays exponentially, and the second one is a $\delta$-pulse, by suitably selecting the duration of the first pulse and the time interval between pulses, the hole can be narrower than the one obtained under stationary burning conditions. 